> Le stress prend des formes très variées, allant de bénéfique, bénigne à traumatique. Chaque individu avec son patrimoine génétique et épigénétique et ses mémoires émotionnelles singulières réagit différemment face au stress. L'effet du stress aigu ou chronique est objectivé par l'élévation d'hormones, comme le cortisol, et d'autres molécules circulantes, évoluant au cours du temps. Après avoir décrit les comportements face au danger, nous exposons dans cette Synthèse, les différentes régulations anatomiques et physiologiques susceptibles de varier lors du passage d'un stress adaptable à un stress traumatique (et de ses mémoires), pouvant entraîner l'installation de troubles de stress post-traumatique (TSPT). Des traitements médicamenteux et des thérapies novatrices permettent d'initier l'extinction des mémoires associées à la peur et d'améliorer la prise en charge des troubles de stress post-traumatiques. <

Le stress, souvent nommé «mal du $x \mathrm{xl}^{\mathrm{e}}$ siècle», prend des formes très variées, allant de bénéfique, bénigne à traumatique. Dans son expression la plus simple, l'apparition d'un évènement soudain, «le stresseur», d'intensité variable, induit une séquence d'évènements adaptatifs engageant le corps et le cerveau pour permettre à la personne de sortir d'une situation jugée dangereuse [1] $(\rightarrow)$.

$(\rightarrow)$ Voir la Synthèse de M.P. Moisanet M. Le Moal, $m / s n^{\circ} 6-7$, juin-juillet 2012, page 612

Ce danger, réel ou supposé, déclenche la peur, cette émotion d'alerte adaptative généralement bénéfique à la survie de l'espèce et de l'individu. Le stress est également une tension, un processus dynamique d'interactions de la personne avec le stresseur et l'environnement. Ce stress peut entraîner des réactions biologiques, psychiques et sociales. Si le stresseur est perçu par la personne comme un danger pour son intégrité physique et/ou psychique (ou celle d'une autre personne), la peur ressentie peut devenir traumatique avec l'acquisition de mémoires associées à la peur.

Vignette (Photo @ Catherine Verney).

\section{Anatomie et physiologie du stress traumatique}

Catherine Verney ${ }^{1-3}$, Pierre Gressens ${ }^{1,2}$, Tania Vitalis ${ }^{1,2}$

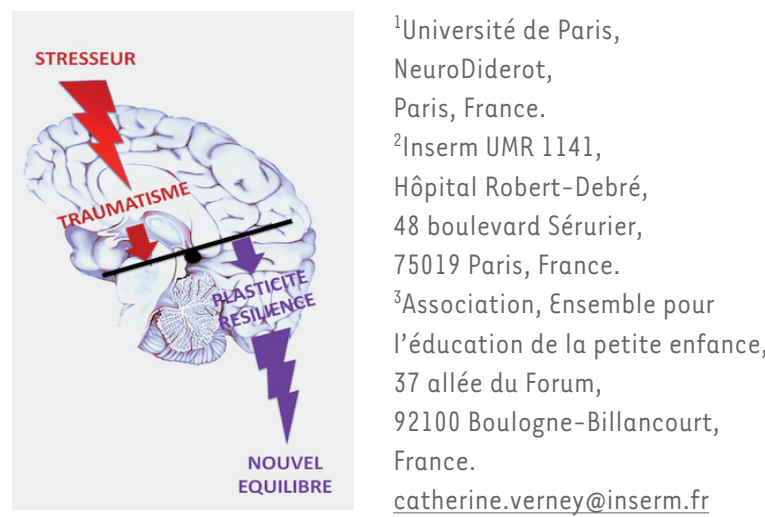

Au cours du temps, certains retrouveront leur équilibre physiologique et psychique. Pour d'autres, l'installation de mémoires traumatiques pourra induire des troubles de stress post-traumatiques (TSPT), comme ceux observés après un attentat. Des dysfonctionnements similaires peuvent être observés lors de stress se répétant ou se prolongeant. Certains auront une réponse «adaptée», non pathologique, alors que d'autres auront une réponse non «adaptée», pathologique, avec toutes les variations entre ces deux extrêmes [2]. Nous ne sommes en effet pas égaux face au stress, qu'il soit traumatique ou chronique [3]. Nos mémoires émotionnelles singulières, nos différences génétiques et épigénétiques, ainsi que nos capacités variables de plasticité cérébrale, sont parmi les paramètres susceptibles de moduler notre réaction, plus ou moins importante face au danger, ainsi que notre capacité de résilience.

\section{Les réactions au stress, les invariants de l'animal à l'homme}

Les réponses comportementales à la peur, suivie de stress face à un danger sont bien conservées chez l'ensemble des vertébrés (Figure 1). Parmi les très nombreux travaux analysant les réactions face au danger, deux séries d'expériences réalisées chez le rat dans les années 1970, nous permettent de présenter les réactions comportementales, physiologiques et les réseaux neuronaux mobilisés chez les mammifères, I'homme compris.

\section{Première série d'expériences}

Ces expériences, réalisées par Henri Laborit [4], dont un film métaphorique a également été réalisé ${ }^{1}$, présentent les réactions à un stress conditionné aigu puis chronique.

\footnotetext{
Resnais A, Laborit H. Mon oncle d'Amérique ; 1980.
} 


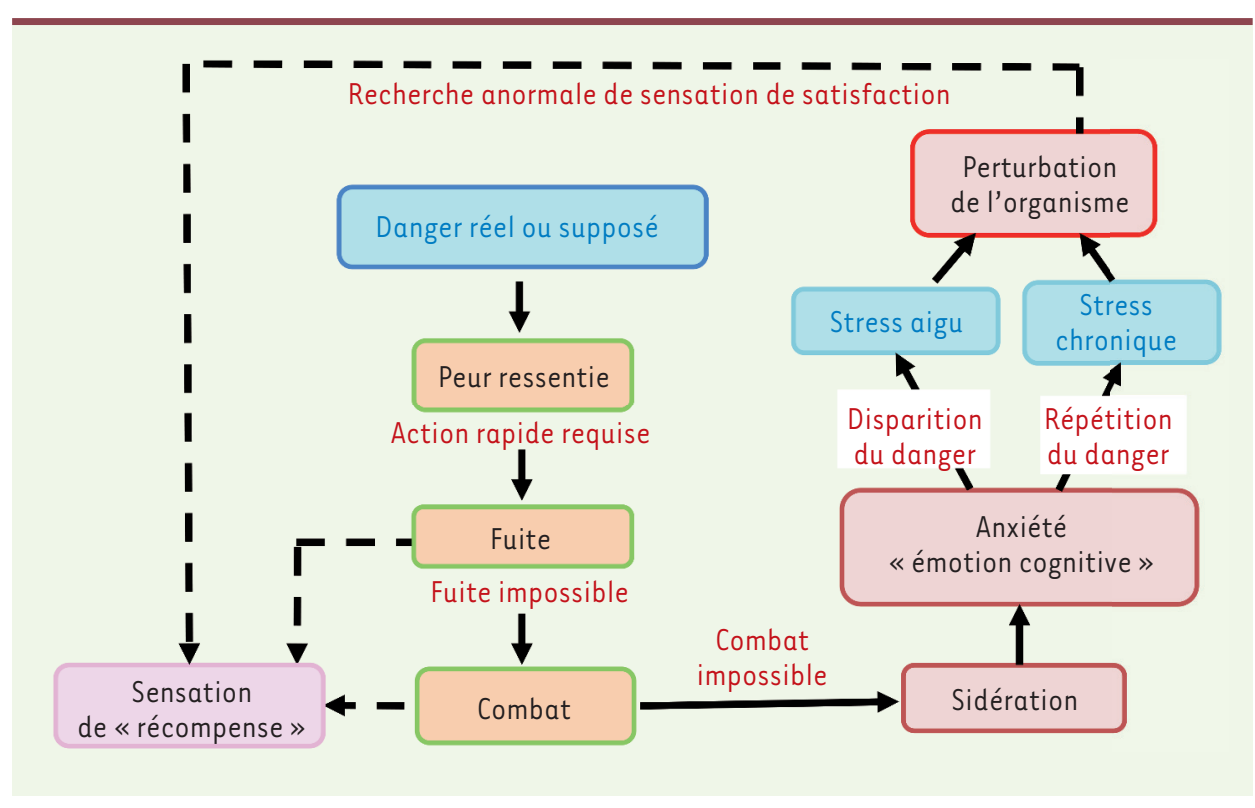

Figure 1. Séquence de comportements face à un danger réel ou supposé. Lorsque l'évitement du danger par l'action dans la fuite ou le combat est impossible, il y a sidération (inhibition de l'action) avec anxiété. L'action dans la fuite et le combat donne une sensation de plaisir, de récompense. Des perturbations de l'organisme peuvent être observées, que ce soit après un stress aigu, limité dans le temps, ou lors d'un stress chronique qui se répète. Ces perturbations peuvent entraîner une recherche anormale de satisfaction, voire une addiction.

Expérience 1 : dans une cage à deux compartiments, un rat subit un choc électrique - précédé d'un son - à travers le plancher de sa cage ; il fuit vers l'autre compartiment de la cage resté ouvert et s'adapte à ce stress aigu.

Expérience 2 : deux rats subissent un stress identique mais ils ne peuvent s'enfuir. Leurs réactions consistent alors en une lutte défensive. Ils sont parfois blessés, mais reprennent ensuite leur vie normalement.

Dans ces deux expériences de stress aigu (limité dans le temps), les rats s'adaptent au stress en s'engageant dans l'action, soit par la fuite, soit par le combat (Figure 1).

Expérience 3 : un rat est dans une cage et reçoit des chocs électriques - précédés d'un son - 10 minutes par jour, pendant 8 jours. II subit ce stress répétitif (chronique) sans pouvoir fuir ; il reste alors à l'arrêt : il y a inhibition de l'action. Ce comportement de sidération ou «freezing » est un comportement de survie, connu dans la nature chez les animaux poursuivis par un prédateur [5]. Ce «freezing» peut être induit par des évènements de dangers très variés et ne présage pas des conséquences à venir.

Dans ces expériences, l'association d'un stimulus neutre (ici un son) à un choc électrique (stimulus douloureux) induit un réflexe conditionné. C'est la réponse automatique (pavlovienne) induite par un stimulus neutre [6]. L'extinction de la réponse conditionnée se produit lorsque le stimulus neutre est présenté souvent mais sans le stimulus douloureux.

Dès les années 1930, Selye avait défini un modèle de gestion du stress en trois phases: une phase d'alarme suivit d'une phase d'adaptation, lorsque l'agent stresseur se prolonge, et d'une phase d'épuisement, quand, au cours du temps, le seuil d'adaptation de l'organisme est dépassé [7]. Même si ce modèle a été largement remanié par l'ajout des réactions émotionnelles et cognitives, les différentes phases physiologiques correspondent à celles observées lors des expériences de Henri Laborit. Le signal d'alarme est donné par le locus cœruleus du tronc cérébral impliqué dans la vigilance (Figure 2). II est très sensible aux stimulus externes et internes de l'organisme [1]. Les neurones noradrénergiques de la formation réticulée du tronc cérébral activent alors le système autonome sympathique avec la libération d'adrénaline et de noradrénaline. Les multiples fluctuations végétatives, comme la salivation, l'activation de la respiration et du rythme cardiaque, signent l'ancrage des réactions au stress dans l'homéostasie corporelle. En parallèle, l'activation de l'axe hypothalamo-hypophysaire-surrénalien (HHS), élément clef de la réponse biologique de régulation corps-cerveau, induit l'activation d'hormones et la libération de glucocorticoïdes, dont le cortisol (Figure 2). L'action par la fuite ou la lutte, ou son inhibition, est régulée au niveau du complexe amygdalien (AMY) activant le système moteur du tronc cérébral (noyau de la substance grise péri-aqueductale) (Figure 3A) [8]. Quand le danger est passé, les régulations parasympathiques du nerf vague et de la libération des glucocorticoïdes tempèrent l'action sympathique, permettant ainsi le retour à l'état basal. La répétition de chocs électriques chez le rat (expérience 3 de Laborit) provoque la stimulation excessive des récepteurs des glucocorticoïdes fortement exprimés dans l'axe HHS (Figure 2) et dans le système limbique stimulé par le danger (complexe amygdalien, hippocampe et cortex préfrontal médian) (Figure 3). La gestion de la peur et de ses mémoires est au centre de la réaction de l'animal face au danger [9].

\section{Seconde série d'expériences}

Un modèle de stress établi chez le rat dans les années 1970 par Roger D Porsolt et al. a été largement utilisé 


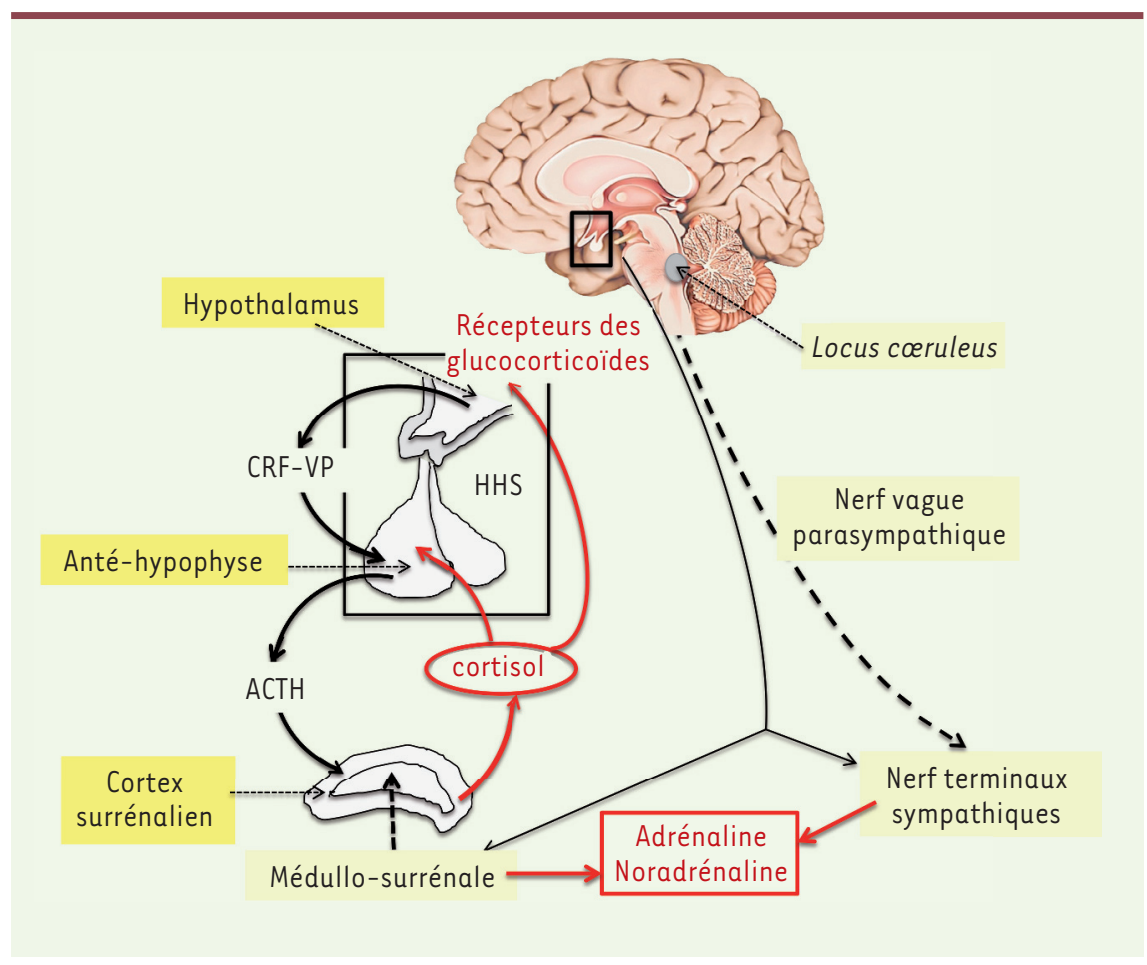

Figure 2. Réponses biologiques à un stress aigu avec l'activation de l'axe corticotrope hypothalamo-hypophysaire-surrénalien (HHS, Encadré). Schématiquement, la libération de $\mathrm{CRH}$ (Corticotropin-releasing hormone) et de vasopressine (VP) hypothalamique active la sécrétion d'ACTH (Adrenocorticotrophin hormone) anté-hypophysaire. L'ACTH circulante induit la libération de cortisol par le cortex surrénalien, qui se fixe sur les récepteurs des glucocorticoïdes hypothalamiques. En parallèle, la CRH active le locus cœruleus, ce qui s'accompagne d'une libération d'adrénaline et de noradrénaline par la médullo-surrénale et par la libération de noradrénaline par les nerfs terminaux sympathiques dans tout le corps. À l'arrêt du stress, la régulation vagale parasympathique tempère l'action sympathique, et l'élévation du cortisol freine, par rétrocontrôle, la sécrétion de CRF et d'ACTH (d'après [1]).

pour tester l'efficacité d'antidépresseurs [10]. Des rats plongés dans un bocal d'eau se mettent à nager puis, après une période de nage vigoureuse, ils se résignent en arrêtant de bouger, avec inhibition de l'action (comme celle observée dans l'expérience 3 précédente). Ils maintiennent la tête hors de l'eau plus ou moins longtemps jusqu'à épuisement. Dans leur capacité à nager plus ou moins longtemps, entrent en jeu d'autres systèmes, comme les systèmes sérotoninergiques et dopaminergiques. Utilisant ce modèle, une série d'expériences ont été réalisées avec des rats de même lignée, ayant subi différentes situations avant le test (100 rats pour chaque expérience) : 1) des rats élevés dans de bonnes conditions : ceux-ci nagent pendant 15 min avant de s'arrêter (et d'être sauvés par l'expérimentateur); 2) des rats qui ont été isolés et privés de leur mère pendant quelques jours à la naissance (ce qui produit des mémoires traumatiques) : ceux-là nagent moins longtemps, pendant $10 \mathrm{~min}$, avant de s'arrêter ; 3 ) des rats élevés dans de bonnes conditions, mais qui subissent le test pendant quelques minutes avant d'être sortis de l'eau, séchés et « rassurés ». Quelques temps après, ces mêmes rats subissent le même test : ils nagent alors pendant 20 min avant de s'arrêter.

Dans la première série d'expérience réalisée par Laborit (Figure 1), les réflexes conditionnés de fuite et de combat stimulent un circuit ancestral, le «conditionnement opérant» avec la sensation de récompense à l'action essentielle à la survie chez pratiquement l'ensemble des vertébrés, du poisson à l'homme [11]. Ce circuit, nommé aussi circuit de renforcement ou de la récompense, est limbique et dopaminergique. Les expériences non conditionnées de Porsolt et al. montrent que les mémoires associées à la peur vécues positivement (expérience 3 ) et la mise en sécurité dans l'enfance, améliorent la motivation à survivre chez le rat. Cette mémoire « intentionnelle», comme la nomme le neuropsychiatre Boris Cyrulnik, est essentielle face à toute situation de danger. Inversement, des expériences traumatiques dans l'enfance (expérience 2 de Porsolt et al.) influent négativement sur les capacités de survie dans la vie adulte. Le circuit «hédonique » de renforcement ou de la récompense est constitué de centres majeurs, comme l'aire tegmentale ventrale - avec la dopamine comme neurotransmetteur - et l'accumbens connectés au complexe amygdalien, au striatum ventral et au cortex préfrontal [12]. Chez l'homme, la stimulation du circuit de récompense méso-cortico-limbique est à la base de la triade «désir - action - satisfaction » particulièrement importante dans l'équilibre psycho-émotionnel de l'individu, dans sa motivation de prise de décision vers l'action au niveau du cortex préfrontal.

Nous ne sommes pas égaux face à l'adversité et un traumatisme peut entraîner chez certains des modifications épigénétiques de l'ADN (comme des méthylations et des modifications de la chromatine) altérant l'expression de certains gènes. Suite à un stress aigu ou chronique, des dérégulations des systèmes dopaminergiques et sérotoninergiques ont été décrites et sont associées à une plus grande susceptibilité à l'anxiété, à la dépression ou à l'addiction [13], même si ces modifications épigénétiques sont en partie réversibles. Des modifications épigénétiques similaires sont aussi observées dans la régulation du cortisol lors de troubles de stress post-traumatique (TSPT) (voir plus loin). 


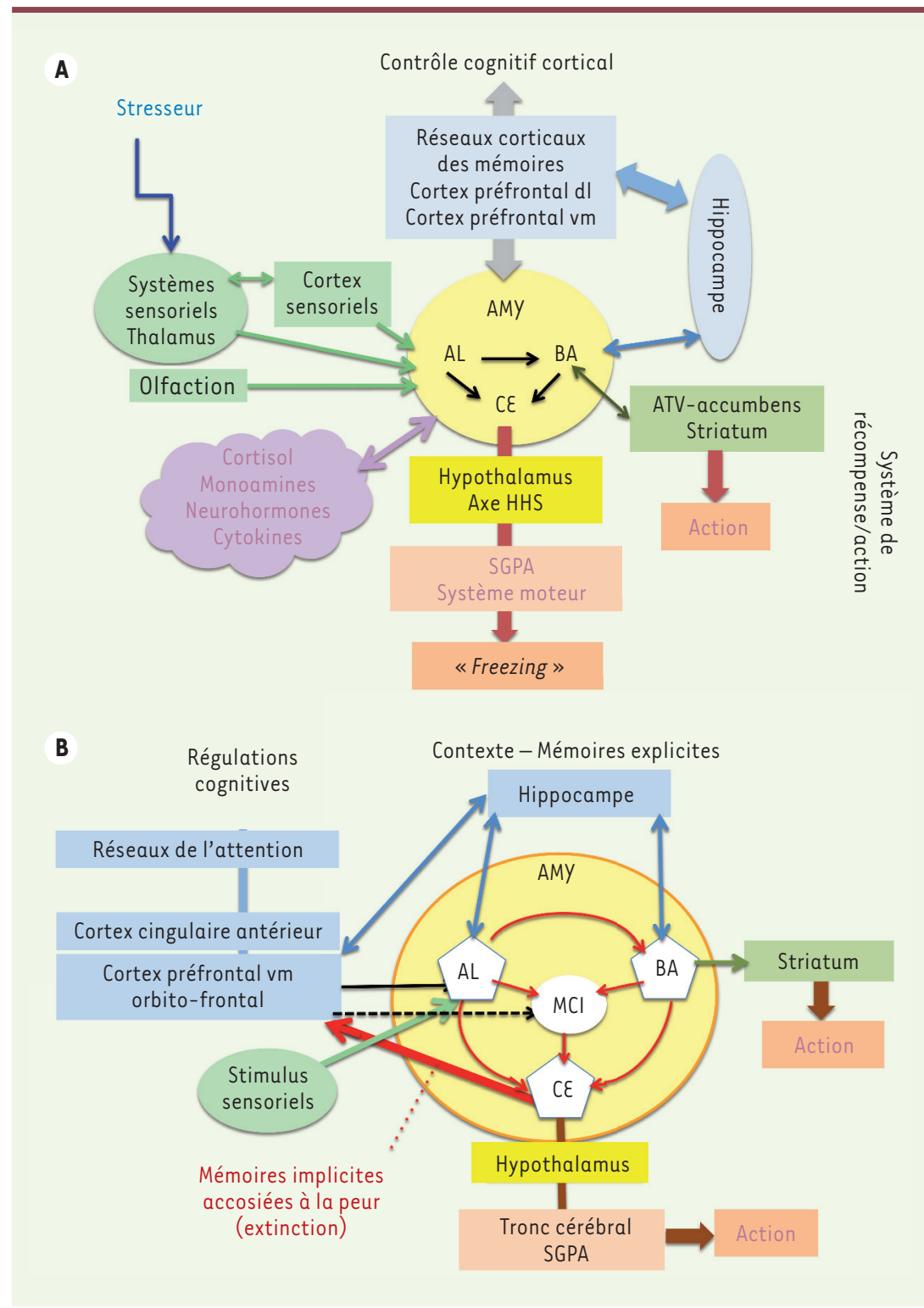

Figure 3. Le complexe amygdalien (AMY, en jaune clair) est au centre du circuit limbique de la peur et de ses mémoires. Les stimulus sensoriels arrivant au niveau de l'AMY déclenchent l'acquisition de la peur. A. Suite à un stresseur (un danger), les informations sensorielles via le thalamus et les cortex sensoriels (en vert clair) convergent vers l'amygdale latérale ( $\mathrm{AL}$ ) puis vers le noyau basal de l'AMY (BA) (acquisition des mémoires de peur), à l'exception des informations olfactives qui arrivent directement à l'AMY sans passer par le thalamus. Le trio AMY-hippocampe-cortex préfrontal ventro-médian $(\mathrm{vm})$ contribue au traitement émotionnel des informations données par le stresseur et gérées par les réseaux corticaux en passant par le cortex préfrontal dorso-latéral (dl) : c'est le control cognitif cortical (en bleu ciel). Le noyau central (CE) de I'AMy régule l'axe hypothalamo-hypophysairesurrénalien (HHS) (en jaune) (voir Figure 2) et le control moteur du tronc cérébral par la substance grise périaqueductal (SGPA), avec l'activation ou l'inhibition de l'action dans le «freezing ». L'activité du BA de l'AMY est régulée par le circuit de récompense/action (valence agréable ou pas du stresseur, en vert foncé). II est composé essentiellement de l'aire tegmentale ventrale (ATV), de l'accumbens et du striatum ventral. Son activation positive mène à l'action. En parallèle, le cortisol, les monoamines, les neurohormones, les cytokines et d'autres molécules circulantes modulent l'ensemble de ces circuits au travers de régulations fines et complexes. $\boldsymbol{B}$. Modèle de circuit régulant les mémoires implicites (en rouge) associées à la peur centrée sur l'AMY. L'expression de la peur correspondrait à une diminution de

l'activité inhibitrice d'interneurones GABAergiques de l'AL et du BA, qui modulerait la voie de sortie de l'AMY via le CE. Lors de la consolidation de la peur, les connexions inhibitrices entre le cortex préfrontal ventro-médian (CPFvm) et les neurones GABA inhibiteurs de la masse cellulaire intercalaire $(\mathrm{MCI})$ se renforcent. L'extinction de la peur serait régulée au niveau du CE en interaction avec le CPFvm (flèche rouge épaisse) et les autres structures mentionnées plus avant (d'après [20]). Les cortex fronto-médians (orbito-frontal et cingulaire antérieur) sont au centre de la réponse émotive/ cognitive au stress régulés par le contexte des mémoires explicites (en bleu). L’AMY régule l'action motrice via le tronc cérébral SGPA (substance grise périaqueductale) et le striatum. Lors de troubles de stress post-traumatiques (TSPT) I'hyperstimulation glutamatergique des noyaux AL et BA peuvent induire l'hypertrophie de l'AMY.

La prise en compte des mémoires traumatiques chez l'homme est relativement récente. II faut attendre les années 1980 pour que les TSPT soient définis cliniquement chez de nombreux anciens combattants américains survivants de la guerre du Vietnam [14]. Actuellement, dans les sociétés occidentales, la prévalence de TSPT est de 5 à $12 \%$, mais ces chiffres pourraient être sous-estimés au vu des circonstances que nous traversons [15]. Toujours dans les années 1980, la découverte par Ledoux et al. du rôle majeur du complexe amygdalien dans la gestion des émotions, dont la peur [16], a été essentielle pour appréhender les composantes psycho-émotionnelles du stress traumatique. Même si les régulations cérébrales sont difficiles à analyser chez l'Homme, les circuits limbiques de la peur, mobilisés lors d'un traumatisme, restent au centre de l'installation des mémoires associées à la peur conduisant ou non à des TSPT. 


\section{Anatomie et physiologie des circuits limbiques et corticaux impliqués dans la réponse au stress}

Chez l'homme, lors d'un stress «adaptable», les informations sensorielles sont transmises en quelques millisecondes au thalamus, au complexe amygdalien-hippocampe-hypothalamus et au cortex préfrontal ventro-médian (CPFvm), ainsi qu'au circuit de la récompense, ce qui permet une évaluation émotionnelle rapide de l'évènement (dangereux ou pas) (Figure 3A). Quelques centaines de millisecondes plus tard, les informations sensorielles sont transmises aux aires corticales cognitives qui, avec l'évaluation du contexte donné par l'hippocampe, permettent une analyse plus fine de la situation. Lors d'un tel stress «adaptable », le traitement cognitif mène à la mobilisation des aires motrices vers l'action [8, 16]. Les mémoires à long terme déclaratives «explicites», gérées par l'hippocampe, participent à l'analyse consciente de la situation avec les mémoires épisodique et sémantique, abondamment décrites dans la littérature [17]. Mais les mémoires émotionnelles mettent en jeu principalement les mémoires «implicites», gérées notamment par le complexe amygdalien : en général, la personne n'en a pas conscience et ne retient pas l'expérience qui en est l'origine [18]. Ces mémoires implicites conduisent à des réactions automatiques de peurs conditionnées et des mémoires d'amorçage perceptif peuvent conduire à l'intrusion dans le conscient de reviviscences, comme observées dans les TSPT [19]. Les mémoires de peurs conditionnées ont été abondamment étudiées chez l'animal [20]. Elles s'installent en différentes phases: l'acquisition (encodage), la consolidation - processus stabilisant l'expérience dans la mémoire à long terme - puis l'extinction, permettant de les surmonter pour aborder un autre apprentissage. Chez l'homme, la plupart des thérapies ont pour but l'extinction des mémoires associées à la peur post-traumatiques afin de les surmonter [2]. Les différentes étapes de peur conditionnée étudiées chez l'animal sont traitées essentiellement par le complexe amygdalien et le cortex préfrontal ventromédian (CPFvm) par ce qui est nommé «l'inhibition amygdalienne »: des régulations complexes entre les réseaux inhibiteurs GABAergiques (gamma-amino butyric acid) et excitateurs glutamatergiques. L'acquisition, la consolidation, l'extinction des mémoires de peur résultent d'interactions entre le complexe amygdalien, l'hippocampe et le cortex préfrontal ventro-médian (Figure $3 B$ ). L'extinction de la peur serait régulée essentiellement au niveau du noyau central $(C \varepsilon)$ du complexe amygdalien en interaction avec le cortex préfrontal ventro-médian [21]. Chez l'homme, les aires corticales gérant les émotions sont beaucoup plus complexes que chez les autres mammifères. Schématiquement, le cortex orbito-frontal est essentiel dans la prise de décision en situation émotionnelle [22], le cortex cingulaire antérieur intègre des souvenirs traumatiques, alors que l'insula serait le récipiendaire des émotions viscérales [21]. La gestion positive du stress, ou simplement son anticipation, induit la stimulation du circuit dopaminergique de renforcement/récompense qui module l'activité des structures cérébrales vers lesquelles il envoie des efférences, comme le complexe amygdalien, l'hypothalamus latéral et les cortex orbitofrontal et cingulaire antérieur [23]. Des recherches récentes suggèrent que les endocannabinoïdes, en interaction avec les systèmes dopaminergiques, pourraient contribuer à l'extinction des mémoires associées à la peur et améliorer les affects négatifs présents dans les TSPT [24]. Les monoamines (noradrénaline, dopamine, sérotonine) jouent des rôles complexes dans la régulation de l'humeur et la gestion d'un stress, tout comme de nombreux neuropeptides (ocytocine), hormones et cytokines [25, 26] (Figure 3A). Chez l'animal, le système immunitaire intervient dans les réponses au stress, dans la lutte ou la fuite $[27](\rightarrow)$.

\section{$(\rightarrow)$ Voir le Forum de C. Jacque et J.M. Thurin, $m / s n^{\circ} 11$, novembre 2002, page 1160}

L'augmentation de la glycémie (hyperglycémie), qui permet la mobilisation rapide d'énergie pour l'action, est ainsi induite, entre autres, par l'IL(interleukine)-6, une cytokine pro-inflammatoire libérée dans le sang, en particulier par les cellules adipeuses brunes [28]. Lors d'un stress psychologique aigu, comme celui produit par un examen, une augmentation des cytokines pro-inflammatoires circulantes (TNF- $\alpha$ [tumor necrosis factor alpha], interféron gamma et IL-6) et une diminution des cytokines anti-inflammatoires (IL-4 et IL-10) sont observées, et sont d'autant plus fortes que l'anxiété induite pendant l'examen est importante [29]. Suite à un stress, toute une gamme de réponses physiologiques, qui dépend de l'histoire de chacun, peut donc être activée pour parfois aboutir à des TSPT.

\section{Dérégulations anatomiques et physiologiques observées lors d'un traumatisme pouvant conduire à des troubles de stress post- traumatique (TSPT)}

Chez l'animal (seconde série d'expériences, voir plus haut), des expériences vécues négativement, telles que des accidents/traumatismes de l'enfance, ou positivement, comme être sain et sauf après avoir subi un accident/traumatisme, orientent significativement la réaction ultérieure face à un nouveau danger. Toute expérience entraîne des stimulations et mémorisations cérébrales avec de continuels remaniements de terminaisons axonales et synaptiques par ce qui est nommé la plasticité cérébrale. Chez l'homme, l'installation de mémoires associées à la peur peut mener à des TPST [2]. Les dérèglements psycho-émotionnels, comme par exemple la reviviscence de scènes traumatiques vécues, provoquent l'hyperstimulation du circuit de la peur (Figure 3 ) et peuvent induire des lésions morphologiques cérébrales. Dans le complexe amygdalien, l'hyperactivité glutamatergique associée à une diminution de l'inhibition GABAergique induit 
une augmentation des arborisations axonales et synaptiques glutamatergiques, entraînant son hypertrophie [30]. L'augmentation du glutamate est aussi observée dans les cibles du complexe amygdalien et l'excitotoxicité provoquée par cette augmentation semble participer aux modifications morphologiques observées dans l'hippocampe: diminution de l'arborisation dendritique et synaptique des neurones des champs ammoniens $C A 3$ et $C A 1^{2}$, et de la neurogenèse hippocampique du gyrus denté [31]. Le cortex préfrontal ventro-médian et le cortex orbito-frontal sont aussi hypotrophiés [21]. Ces changements morphologiques signent la consolidation des mémoires associées à la peur [32]. Le circuit de la peur est connecté aux deux grands réseaux neuronaux décrits comme gérant les hautes fonctions cognitives: principalement celui gérant les fonctions exécutives, activé lors de nos actions quotidiennes (réseaux frontaux-pariétaux) et le réseau cérébral, nommé « réseau du mode par défaut », qui s'active lorsque nous ne faisons rien de particulier (aires corticales médianes et lobe pariétal latéral). Des recherches entreprises récemment tentent d'établir des corrélations entre l'activation de ces réseaux et les différents symptômes observés chez les patients victimes de TSPT [26]. En clinique, les symptômes de dérégulations émotionnelles observées lors de TSPT oscillent entre deux extrêmes [33] : 1) un manque d'expression des émotions avec des reviviscences subjectives, ou flash backs, associées à une hyperexcitation. Au niveau cérébral, il y aurait un déficit d'inhibition préfrontal des régions limbiques préfrontales ventromédiales avec une hyperactivation du complexe amygdalien, associée à une hyperactivité de la partie rostrale du cortex cingulaire antérieure, récipiendaire des mémoires traumatiques à long terme; 2) une dissociation avec la surexpression des émotions. Cela correspondrait à une inhibition cortico-limbique excessive incluant la partie dorsale du cortex cingulaire antérieur et le cortex préfrontal médian. On observe une sorte de désengagement subjectif du contenu émotionnel de la mémoire traumatique (avec dépersonnalisation ou déréalisation) reposant sur l'inhibition préfrontale médiane des régions limbiques (complexe amygdalien, insula, cortex cingulaire antérieur).

Comme nous l'avons mentionné, nous ne sommes pas égaux face à un danger, avec des régulations épigénétiques touchant les systèmes dopaminergiques et sérotoninergiques induisant des vulnérabilités singulières [13]. Notre singularité s'exprime également dans nos différentes réponses au stress - en particulier psychologique - reliées à d'importantes différences de régulation du cortisol $[3,26]$. La modulation de la réponse au cortisol est au centre de nombreuses boucles régulatrices tant génétiques qu'épigénétiques. Lors de l'arrivée massive de cortisol, les récepteurs des glucocorticoïdes sont internalisés dans le noyau cellulaire et modulent l'expression de différents gènes, comme FKBP5 (FK506binding protein 51), qui code une protéine qui réduit l'affinité entre le cortisol et ses récepteurs [34]. La méthylation de l'ADN diminue l'expression de la protéine FKBP5 et est associée à une moindre réaction au stress. Récemment, il a été montré que ces méthylations pouvaient être corrélées à différentes formes d'expression cognitive de TSPT associées à des altérations cérébrales, comme une hypotrophie hippocampique

\footnotetext{
${ }^{2}$ L'hippocampe est formé des champs ammoniens (CA1, CA2, CA3 et CA4) et du gyrus denté.
}

observée chez certains sujets [35]. D'autres altérations épigénétiques ont été détectées touchant la régulation du gène BDNF (brain-derived neurotrophic factor), essentiel à la plasticité cérébrale [36]. Certaines de ces modifications sont utilisées comme marqueurs prédictifs de TSPT ou de leur guérison, quand le taux de méthylation revient à la normale [37]. C'est le cas du gène ZFP57 (zinc finger protein 57) dont le taux de méthylation, dans les cellules sanguines, est diminué chez les soldats qui ont été traités par psychothérapie [38].

L'état de stress post-traumatique est par ailleurs associé à une modulation complexe de l'immunité, humorale et cellulaire $[28,39]$. Les taux sanguins de cytokines pro-inflammatoires (IL-1 $\beta, \mathrm{IL}-6, \mathrm{TNF}-\alpha$ ) et de CRP (protéine $C$-réactive) sont plus élevés chez les individus souffrant de TSPT. La chronicité du stress et son étendue dans le temps, induisent également, progressivement, une modification de l'immunité cellulaire, avec un transfert d'une réponse lymphocytaire de type THl à une réponse de type $\mathrm{TH} 2$, conduisant à une moins bonne immunité cellulaire $(\mathrm{TH})$ et à une augmentation du seuil de déclenchement des réactions allergiques ( $\mathrm{TH} 2)$ [29]. De plus en plus d'études mentionnent le stress comme un co-facteur décisif dans le déclenchement de maladies comme les maladies cardio-vasculaires, dermatologiques, dégénératives et les cancers (cette liste n'est pas exhaustive). D'où l'importance de prendre en compte la gestion du stress dans la prévention et le traitement de ces maladies [39].

\section{Les voies vers la guérison d'un stress traumatique}

Face au stress de la vie quotidienne, nous pouvons agir sur la respiration, seule fonction végétative pouvant être régulée consciemment tout en restant autonome [40]. Une respiration lente permet en effet de réguler un stress par l'homéostasie parasympathique vagale et la régulation de l'axe corticotrope (HHS) (Figure 2 et Figure 4). Différentes techniques (relaxation, sophrologie, cohérence cardiaque, yoga, méditation, etc.), dont certaines sont appliquées par les professionnels de santé, explorent l'intéroception - la prise de conscience de nos ressentis -, essentielle dans la gestion de nos émotions et de notre stress [41, 42]. Ces pratiques entraînent une diminution du cortisol sanguin et la régulation de la production de différentes hormones et neurotransmetteurs, comme la sérotonine ou la dopamine, modulateurs de l'humeur [14, 27, 33]. La connaissance et la maîtrise de certaines de ces pratiques permet à la personne une meilleure gestion de son stress, voire de son traumatisme. 


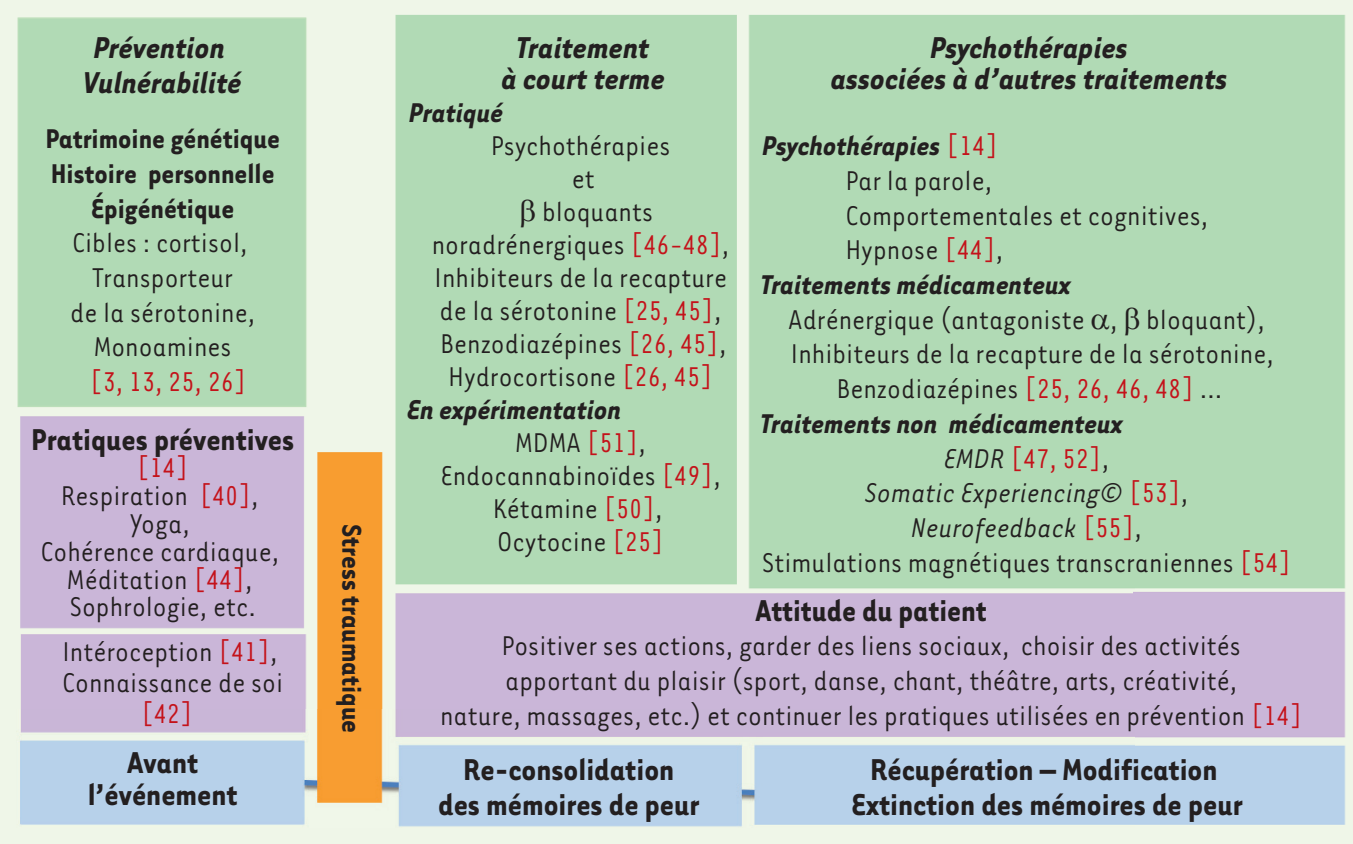

Figure 4. Étiologie et traitements de troubles de stress post-traumatique (TSPT). Avant l'évènement traumatisant (première colonne), il est nécessaire de faire de la prévention (fond vert). Notre capacité à surmonter un stress dépend de notre patrimoine génétique et de notre histoire personnelle ayant entraîné des modifications épigénétiques pouvant modifier notre vulnérabilité à un nouveau stress. Différentes pratiques préventives (fond violet) fondées sur la respiration nous permettent d'explorer notre intéroception et d'approfondir notre connaissance de soi afin d'affiner l'analyse de nos réactions au stress. Après le traumatisme (deuxième colonne), les mémoires associées à la peur ne sont pas stabilisées et nécessitent d'être consolidées (fond bleu). Des thérapies doivent être associées. Certaines sont en cours d'expérimentation comme le MDMA (3,4 méthylènedioxy-méthamphétamine). Pour traiter des TSPT (troisième colonne, fond vert), des psychothérapies seront associées à d'autres traitements médicamenteux et/ou non médicamenteux, comme l'EMDR (eye-movement desensitization reprocessing). Ces thérapies agissent sur la plasticité cérébrale par récupération, modification, et par extinction des mémoires associées à la peur (fond bleu). Le patient choisit des activités qui lui font plaisir, en positivant et en développant des liens sociaux (fond violet).

Les patients avec des troubles de stress post-traumatique (TSPT) peuvent présenter des dérégulations physiologiques et des modifications anatomiques cérébrales - que l'on observe en imagerie fonctionnelle [43] - signant l'installation de mémoires associées à la peur $[20,26]$. En psychologie clinique, les symptômes de dérégulations émotionnelles oscillent entre une hyperexcitation, avec des reviviscences subjectives, et une inhibition, souvent associées à des dissociations, à des difficultés de concentration et à un déficit relationnel [33]. Submergé par la résurgence de mémoires associées à la peur, le patient ne parvient plus à penser la situation et à vivre sa vie (Figure 4). Des thérapies (psychothérapies, thérapies cognitives comportementales, hypnose) aident à contenir la consolidation de souvenirs délétères $[33,44]$. Des traitements médicamenteux, comme les $\beta$-bloquants noradrénergiques, l'hydrocortisone, et des inhibiteurs de recapture de la sérotonine, ont été largement utilisés pour aider des personnes victimes de catastrophes et prévenir la survenue de TSPT [45]. Intervenir juste après l'événement traumatique - avant son installation dans la mémoire à long terme - active significativement les capacités de résilience des patients. La prise de $\beta$-bloquants noradrénergiques (propanolol), juste avant une séance de psychothérapie avec la remémoration de l'événement traumatique, permet, après six semaines de traitement, une amélioration significative chez $70 \%$ des patients [46]. Le propanolol inhibe la consolidation des mémoires en bloquant les effets de la noradrénaline cérébrale. II participe à diminuer la valence traumatique des mémoires lorsque celles-ci sont revisitées au cours d'une séance. Après les attentats de 2015, en France, Brunet et al. ont associé le propanolol à I'EMDR (eye-movement desensitization reprocessing, en français, désensibilisation et reprogrammation par des mouvements oculaires) avec des résultats prometteurs toujours en cours d'évaluation [47, 48]. D'autres molécules ciblant d'autres systèmes, comme les endocannabinoïdes, la kétamine, l'ocytocine [25, $49,50]$, en cours d'essais cliniques, pourraient être utilisées à la place du propanolol. Récemment le MDMA (3,4 méthyl enedioxy méthamphétamine), utilisé en 
association à une psychothérapie, a donné des résultats prometteurs qui devront être confirmés (Figure 4) [51].

Les lésions cérébrales observées chez certains patients présentant des TSPT sévères sont induites par l'hyperactivation des circuits limbiques (hypertrophie du complexe amygdalien) associée à une inhibition/ diminution des fonctions exécutives de l'action (hypotrophie préfrontale) [43]. Les différentes thérapies fondées sur la stimulation de la plasticité cérébrale ont pour but de surmonter les mémoires traumatiques implicites, invalidantes, en agissant sur les différentes étapes, récupération, modification, extinction (Figure 4). En communiquant par une parole empathique et positive et en utilisant les voies d'entrées sensorielles et motrices, il est possible de revisiter les circuits limbiques et les mémoires traumatiques par la plasticité cérébrale. Par exemple, l'EMDR revisite les circuits de mémoires traumatiques par stimulation droite/gauche alternée des yeux, alors que le patient se remémore le traumatisme avec l'aide du thérapeute [52]. Le somatic experiencing@ (en français, expérience somatique) fondé sur l'expression des émotions par le corps, permet de libérer des informations sensorielles figées dans le cerveau - comme la colère - et d'activer chez la personne ses capacités de résilience [14, 53].

L'étude des synchronisations cérébrales des ondes thêta $(4 \text { à } 8 \mathrm{~Hz})^{3}$ présentes dans le circuit limbique, ouvre des voies thérapeutiques non invasives par stimulations cérébrales. Des stimulations magnétiques transcrâniennes intermittentes de faible fréquence (thêta, alpha) pratiquées en milieu hospitalier, améliorent des symptômes de TPST par réactivation de la mémoire de travail ${ }^{4}$ chez les patients [54].

Le neurofeedback (rétroaction) permet à la personne de modifier son activité cérébrale grâce à l'encéphalographie visualisée en temps réel (diminution de la fréquence des ondes cérébrales). Cet entraînement induit de nouvelles associations permettant un remodelage des mémoires traumatiques [55].

Dans ce panel de traitements disponibles, les thérapies nécessitent d'être sélectionnées afin de traiter les symptômes de TPST spécifiques à chaque patient [33]. Un traitement médicamenteux doit toujours être accompagné de thérapies psychocorporelles, car l'objet principal de toute thérapie est de rendre modulables (plastiques) les mémoires traumatiques du patient, de les revisiter afin de les lui rendre acceptables. Comme le dit Boris Cyrulnik, la résilience n'est pas le retour à la vie d'avant, mais la découverte d'un nouvel équilibre de vie post-traumatique [56]. Pour chaque patient (Figure 4), entreprendre des activités qui lui plaisent, créer des liens sociaux et positiver ses actions contribuent largement à guérir les cicatrices du traumatisme et à installer un nouvel équilibre [14]. $\diamond$

\footnotetext{
${ }^{3}$ Ondes aux fréquences assez basses $(4-8 \mathrm{~Hz})$, produites lors de la première phase du sommeil lent (l'endormissement) ou dans un état de profonde relaxation.

${ }^{4}$ La mémoire de travail (ou mémoire à court terme) est la mémoire du présent. Elle permet de manipuler et de retenir des informations pendant la réalisation d'une tâche ou d'une activité. Elle fonctionne comme une mémoire tampon: les informations qu'elle véhicule peuvent être rapidement effacées, ou stockées dans la mémoire à long terme.
}

\section{SUMMARY}

Anatomy and physiology of traumatic stress

Facing a more or less intrusive stress, some individuals can cope as they are more resilient, while others get traumatized and further develop a Post Traumatic Stress Disorder (PTSD). Individuals are not equal facing traumatic stress for genetic/epigenetic or personal reasons. This review analyzes from animal models to human, the neurobiological changes detected when the stress switch from adaptable in everyday life to pathological leading to PTSD. Fear memories lead to the disruption of the anatomy/morphology of emotional-memory networks centered on the amygaloïd complex and hippocampal hub associated with the homeostatic unbalance of the body-brain exchanges of molecules such as hormones, neuromodulators or peptides. Persistent fear memories are hardly handled by the frontal ability for decision making towards action. But these fear memories can be revisited by different therapies recruiting cerebral plasticity and resilience. Current understanding of PTSD allowed to develop a series of efficient treatments associating precise medicine to diverse body-mind therapies. $\diamond$

\section{LIENS D'INTÉRÊT}

Les auteurs déclarent n'avoir aucun lien d'intérêt concernant les données publiées dans cet article.

\section{RÉFÉRENCES}

1. Moisan MP, Le Moal M. Le stress dans tous ses états. Med Sci (Paris) 2012 ; $28: 612-7$

2. Mary A, Dayan J, Leone $G$, et al. Resilience after trauma: the role of memory suppression. Science $2020 ; 367$ : eaay8477.

3. Argentieri MA, Nagarajan S, Seddighzadeh B, et al. Epigenetic pathways in human disease: the impact of DNA methylation on stress-related pathogenesis and current challenges in biomarker development. EBioMedicine 2017 ; 18 : 327-50.

4. Laborit H. L'éloge de la fuite. Paris : Folio Essais, 1976 : 188 p.

5. Roelofs K. Freeze for action: neurobiological mechanisms in animal and human freezing. Phil Trans R Soc 2017 ; B 372 : 20160206.

6. Rehman I, Mahabadi N, Sanvictores T, et al. Classical conditioning. Treasure Island (FL) : StatPearls Publishing,2021.

7. Selye H. Stress and general adaptation syndrome. Br Med J $1950 ; 17$ : 1383-92.

8. LeDoux JE, Pine, DS. Using neuroscience to help understand fear and anxiety: a two-system framework. Am J Psychiatry 2016; 173: 1083-93.

9. LeDoux J. Rethinking the emotional brain. Neuron $2012 ; 73: 653-76$.

10. Porsold RD, le Pichon M, Jalfre M. Depression: a new animal model sensitive to antidepressant treatments. Nature $1977: 266: 730-32$.

11. O'Connell LA, Hofmann HA. The vertebrate mesolimbic reward system and social behavior network: a comparative synthesis. J Comp Neurol 2011 ; 519 : 3599-639.

12. Thierry AM, Tassin JP, Blanc G, et al. Selective activation of the mesocortical dopaminergic system by stress. Nature $1976 ; 263: 242-44$.

13. Douma $\varepsilon H$, de Kloet $\varepsilon R$. Stress-induced plasticity and functioning of ventral tegmental dopamine neurons. Neurosci Biobehav Rev 2020 ; $108: 48-77$.

14. Der Kolk BV. Le corps n'oublie rien. Le cerveau, l'esprit et le corps dans la guérison du traumatisme. Paris : Albin Michel, 2018 : 590 p.

15. Inserm. Dossier-Information, troubles-stress-post-traumatique. Paris : Éditions Inserm, 2020.

16. LeDoux JE, Iwata J, Cicchetti P, et al. Different projections of the central amygdaloid nucleus mediate autonomic and behavioral correlates of conditioned fear.J Neurosci $1988 ; 8: 2517-29$. 


\section{RÉFÉRENCES}

17. inserm.fr/information-en-sante/dossiers-information/memoire

18. Lejeune A, Delage M. La mémoire sans souvenir. Paris : Odile Jacob, 2017 : 336 p.

19. Van Elzakker MB, Dahlgren MK, Davis FC, et al. From Pavlov to PTSD: the extinction of conditioned fear in rodents, humans, and anxiety disorders. Neurobiol Learn Mem 2014 ; 113 : 3-18.

20. Ressler RL, Maren S. Synaptic encoding of fear memories in the amygdala. Curr Opin Neurobiol $2019 ; 54: 54-9$.

21. LeDoux JE, Brown R. A higher-order of theory of emotional consciousness. Proc Natl Acad Sci USA 2017 ; 15 : ع2016-25.

22. Damasio AR. L'erreur de Descartes. Paris : Odile Jacob, 2010 : 396 p.

23. Berger $B$, Gaspar $P$, Verney C. Dopaminergic innervation of the cerebral cortex: unexpected differences between rodents and primates. Trends Neurosci $1991 ; 14: 21-7$.

24. Ney LJ, Akhurst J, Bruno R et al. Dopamine, endocannabinoids and their interaction in fear extinction and negative affect in PTSD. Prog Neuropsychopharmacol Biol Psychiatry 2021; 105 110118.

25. Vitalis T, Verney C. Roles of the serotoninergic system in coping in traumatic stress. Intechopen $2021 ; 10.5772: 69000$.

26. Yehuda, R., Hoge, C, McFarlane A, et al. Post-traumatic stress disorder. Nat Rev Dis Primers 2015 ; $1: 15057$

27. Jacque C, Thurin JM. Stress, immunité et physiologie du système nerveux. Med Sci (Paris) 2002 ; $18: 1160-6$.

28. Ping $H$, Desrouleaux R, Israni-Winger $K$, et al. Origin and function of stress-induced IL-6 in murine models. Cell $2020 ; 182: 372-87$

29. Maes $M$, Song C, Lin A, et al. The effects of psychological stress on humans: increased production of pro-inflammatory cytokines and a Thl-like response in stress-induced anxiety. Cytokine 1998 $10: 313-8$.

30. Zhang JY, Liu TH, He Y, et al. Chronic stress remodels synapses in an amygdala circuit-specific manner. Biol Psychiatry $2019 ; 85$ : 189-201.

31. Stein-Behrens B, Mattson MP, Chang I, et al. Stress exacerbates neuron loss and cytoskeletal pathology in the hippocampus. J Neurosci $1994 ; 14: 5373-80$.

32. Gagnepain P, Hulbert J, Anderson MC. Parallel regulation of memory and emotion supports the suppression of intrusive memories. J Neurosci 2017 ; 37 : 6423-41.

33. Lanius RA, Vermetten $\varepsilon$, Loewenstein RJ, et al. Emotion modulation in PTSD: clinical and neurobiological evidence for a dissociative subtype. Am J Psychiatry $2010 ; 167: 640-47$

34. Zannas A, Wiechmann T, Gassen N, et al. Gene-stress-epigenetic regulation of FKBP5: clinical and translational implications. Neuropsychopharmacology $2016 ; 41: 261-74$.

35. Yun JY, Jin MJ, Kim S, et al. Stress-related cognitive style is related to volumetric change of the hippocampus and FK506 binding protein 5 polymorphism in post-traumatic stress disorder. Psychol Med $2020 ; 7: 1-12$

36. Notaras $M$, van den Buuse M. Neurobiology of BDNF in fear memory, sensitivity to stress, and stress-related disorders. Mol Psychiatry $2020 ; 25: 2251-74$.

37. Boks MP, Rutten BP, Geuze $\varepsilon$, et al. SKA2 methylation is involved in cortisol stress reactivity and predicts the development of post-traumatic stress disorder (PTSD) after military deployment. CH Neuropsychopharmacol $2016 ; 41: 1350-56$.

38. Vinkers CH, Geuze $\varepsilon$, van Rooij SJH, et al. Successful treatment of post-traumatic stress disorder reverses DNA methylation marks. Mol Psychiatry 2019; 10 : 1038.

39. Duric V, Clayton S, Leong ML, et al. Comorbidity factors and brain mechanisms linking chronic stress and systemic illness. Neural Plast $2016: 5460732$.
40. Berthoud HR, Neuhuber WL. Functional and chemical anatomy of the afferent vagal system. Auton Neurosci $2000 ; 85: 1-17$.

41. Schulz A, Vögele C. Interoception and stress. Front Psychol $2015 ; 6: 993$.

42. Damasio AR. Le sentiment même de soi : corps, émotions, conscience. Paris, Odile Jacob, $1999: 480 p$

43. Kamiya K, Abe 0 . Imaging of posttraumatic stress disorder neuroimaging. Clin North Am 2020 ; 30 : 115-23.

44. Lynn SJ, Malakataris A, Condon L, et al. Post-traumatic stress disorder: cognitive hypnotherapy, mindfulness, and acceptance-based treatment approaches. Am J Clin Hypn 2012 ; 54 : 311-30.

45. Sijbrandij M, Kleiboer A, Bisson Jl, et al. Pharmacological prevention of posttraumatic stress disorder and acute stress disorder: a systematic review and meta-analysis. Lancet Psychiatry $2015 ; 2: 413-21$.

46. Brunet A, Saunier D, Liu A, et al. Reduction of PTSD symptoms with prereactivation propranolol therapy: a randomized controlled trial. Am J Psychiatry $2018 ; 175: 427-33$.

47. Wilson G, Farrell D, Barron I, et al. The use of eye-movement desensitization reprocessing (EMDR) therapy in treating post-traumatic stress disorder: a systematic narrative review. Front Psychol $2018 ; 9: 923$

48. Brunet A, Ayrolles L, Gambotti R, et al. Paris MEM: a study protocol for an effectiveness and efficiency trial on the treatment of traumatic stress in France after the 2015-16 terrorist attacks. BMC Psychiatry 2019; 19 : 351.

49. Bitencourt RM, Takahashi RN. Cannabidiol as a therapeutic alternative for post-traumatic stress disorder : from bench research to confirmation in human trials. Front Neurosci $2018 ; 24 ; 2$ : 502 .

50. Abdallah CG, Roache JD, Averill LA, et al. Repeated ketamine infusions for antidepressant-resistant PTSD: methods of a multicenter, randomized, placebo-controlled clinical trial. Contemp Clin Trials $2019 ; 81: 11-8$.

51. Vermetten $\varepsilon$, Yehuda R. MDMA-assisted psychotherapy for posttraumatic stress disorder: a promising novel approach to treatment. Neuropsychopharmacology $2020 ; 45: 231-2$.

52. Shapiro F. Se libérer des souvenirs traumatisants avec l'EMDR. Paris : Points Essais, 2018 : 384 p.

53. Levine PA. Guérir par-delà les mots. Comment le corps dissipe le traumatisme et restaure le bien-être. Paris : Interદ́ditions, 2020 : 432 p.

54. Philip NS, Barredo J, Aiken $\varepsilon$, et al. Theta burst transcranial magnetic stimulation for posttraumatic stress disorder.J Psychiatry 2019; 176: 939-48.

55. Leem J, Cheong MJ, Yoon SH, et al. Neurofeedback self-regulating training in patients with Post traumatic stress disorder: AA randomized controlled trial study protocol. Integrat Medicine Res $2020 ; 9$ : 100464.

56. Cyrulnik B. Sous le signe du lien. Paris : Hachette, 1989. 320 p.

\section{LA FONDATION PREMUP : UN OPÉRATEUR DE TERRAIN EN PÉRINATALITÉ RECONNU POUR SON EXCELLENCE ET SON INTERDISCIPLINARITÉ}

La Fondation de coopération scientifique PremUp, unique en Europe, intervient sur la prévention du handicap à la naissance, par la protection de la santé de la femme enceinte et du nouveau-né.

TIRÉS À PART

C. Verney

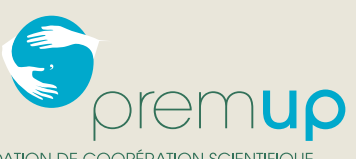

SUR LA GROSSESSE ET LA PRÉMATURITE

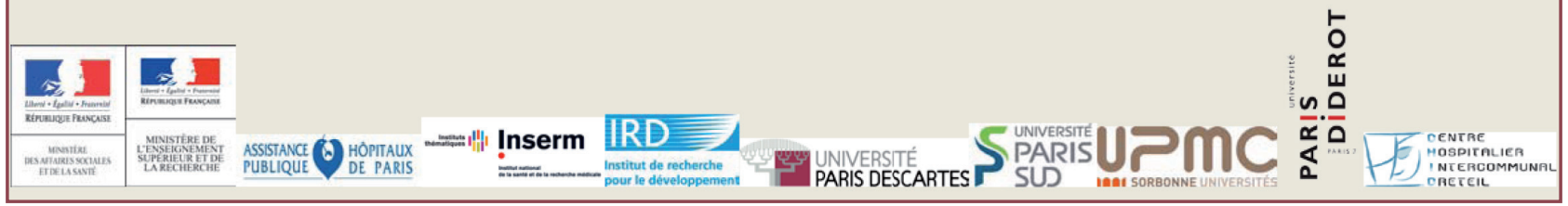

